\title{
Der mikroskopische und kulturelle Nachweis der Tuberkelbazillen im Blasen- und Nierenharn bei Nierentuberkulose.
}

\author{
Von \\ Kannosuke Kon. \\ (今 官之助)
}

(Aus der chirurgischen Klinik der Universität, Sendai.

Direktor: Prof. Dr. M. Muto.)

(Received for publication, March 1, 1945)

Zur Feststellung der Tuberkelbazillen im Harn ist das kulturelle Verfahren aus verschiedenen Gründen noch nicht in allgemeinen Gebrauch gekommen, während der mikroskopische Nachweis dieser Krankheitserreger im Ausstrich des Harnsediments schon von manchen Praktikern ausgeführt worden ist. .Da ich die beiden genannten Verfahren an ein und demselben Sediment des Blasen-und des durch Ureterenkatheterismus getrennt aufgefangenen Nierenharns bei 114 Fällen von Neierentuberkulose durchgeführt habe und glaube, einige klinisch bemerkenswerte Resultate erhalten zu haben, will ich im folgenden kurz darüber berichten.

Zur Mikroskopierung wurden aus dem Harnsediment nach einem von mir modifizierten Verfahren ${ }^{1 l}$ gefärbte Strichpräparate-Färbung mit Ziehl'scher Karbolfuchsinlösung, Entfärbung mit 3\%iger HClAlkohollösung, ohne Nachfärben-hergestellt. Zur Kultivierung der Tuberkelbazillen benutzte ich Oka-Katakura'sche Nährböden') und zwar 3-4 mal für jeden Versuch, nachdem ich das Restsediment mit Schwefelsäure-Isravinlösung vorbehandelt und nochmals stark zentrifugiert hatte..

I. Untersuchungsergebnisse bei Blasenharn.

Bei fast allen 114 Fällen wurde starke Pyurie d.h. Auftreten zahlreicher bzw. zahlloser Eiterkörperchen im Blasenharn beobachtet. Ausnahamsweise war sie nur bei einigen Fällen äusserst schwach, von denen bei der Operation 3 Fälle als geschlossene tuberkulöse Pyonephrose und die anderen 3 als allerfrühestes Stadium von Nierentuberkulose erkannt wurden.

Die Tuberkelbazillen konnten, wie Tabelle I zeigt, mikroskopisch bei 98 Fällen ( $86 \%$ ) und zwar bei 68 mässig oder zahlreich, bei 30 spärlich, und kulturell bei $107(94 \%)$ und zwar, bei 77 sehr reichlich und bei 30 mässig oder spärlich nachgewiesen werden. Die Feststellungshäufigkeit 


\section{TABELLE I.}

Die Ergebnisse der mikroskopischen und kulturellen

Untersuchung des Blasenharns.

Bemg. Die Tuberkelbazillen wurden mikroskopisch zahlreich in jedem Gesichtsfeld (H), vereinzelt aber in Häufchen und Grüppchen, bald stellenweise mässig reichlich (H), vereinzelt in geringer $\mathrm{Zahl}$ aber ohne Mühe $(+)$, oder nur 2 bis einige Stäbchen in ganzen Gesichtsfeld eines Präparates mit Geduld ( \pm ) festgestellt. Die Zahl der Kolonien bei Kultivierung bedeutet die durchschnittliche Werte.

\begin{tabular}{|c|c|c|c|c|c|c|c|c|}
\hline \multirow{2}{*}{\multicolumn{2}{|c|}{ Kolonienzahl }} & \multicolumn{7}{|c|}{ Zahl d. Fälle bei Färbung } \\
\hline & & \# & H & + & \pm & - & & \\
\hline \multirow{6}{*}{ 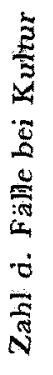 } & unzählbar & 28 & 36 & 7 & 6 & & 77 & \multirow{3}{*}{107} \\
\hline & ca. $10-50$ & 1 & 2 & 6 & 7 & 4 & 20 & \\
\hline & $1-5$ & & 1 & 2 & 2 & 5 & 10 & \\
\hline & - & & & : & & 7 & & 7 \\
\hline & \multirow{2}{*}{ total } & 29 & 39 & 15 & 15 & \multirow[b]{2}{*}{16} & \multirow{2}{*}{\multicolumn{2}{|c|}{114}} \\
\hline & & \multicolumn{4}{|c|}{98} & & & \\
\hline
\end{tabular}

war selbstverständlich grösser beim kulturellen Verfahren als bei der mikroskopischen Untersuchung. Im Vergleich der gefärbten Bazillenmit der kultivierten Kolónienzahl aus demselben Harn war sie im allgemeinen zahlreicher bei der Kultivierung als bei der Mikroskopierung, wäbrend die Kolonienzahl dabei im grossen und ganzen mit der mikroskopisch nachgewiesenen Bazillenzahl parallel ging. So ist die Kultur als Auffindungsverfahren der Tuberkelbazillen sicherer als die mikroskopische Untersuchung, obwohl man ungefähr 3 bis 4 Wochen warten muss, bis die Ergebnisse erkennbar sind. Eine wichtige Ursache für die grössere Zuverlässigkeit der Nachweises durch Kultur liegt nach meiner Erfahrung darin, dass bei der Kultivierung unvergleichlich grössere Mengen Harnsediment-mehr als das einige Hundertfache-als bei der mikroskopischen Untersuchung untersucht werden können.

Nach Wildbolz (1927) ${ }^{3)}$ gelang es früher, nur bei $20 \%$ bis $50 \%$ der Kranken mit Nieren- resp. Blasentuberkulose die Bazillen mikroskopisch nachzuweisen; die Zahl der positiven Bazillenbefunde wuchs aber bei wachsender Sorgfalt und besserer Untersuchungstechnik in dieser Zeit schon so hoch, dass die meisten derzeitigen Autoren positive Befunde bei 80 bis $90 \%$ aller an Harntuberkulose Erkrankten mitteilten. Nach den letzten Angaben einiger Autoren ${ }^{4-7)}$ unseres Landes 'betrug die mikroskopische Feststellungshäufigkeit der Tuberkelbazillen im Blasenharn 42 
bis $70 \%$ aller an Nierentuberkulose Erkrankten. Kitagawa u.a. ${ }^{8)}$ äusserten sich, dass sie $49 \%$ positive Resultate in den anfänglichen 2 Jahren und dann $90 \%$ in den folgenden $3 \mathrm{Jahren}$ ihrer Untersuchungen erzielten. Aus unserer Klinik berichtete Koizumi ${ }^{5}$ von $65 \%$ positiven Bazillenbefunden bei 638 Fällen von Nephrektomie wegen Nierentuberkulose, die im Zeitlauf von 23 Jahren von mehreren Untersuchern geprüft wurden. Jetzt beschäftigte ich mich allein mit diesen Untersuchungen und konnte färberisch $86 \%$ positive Resultate erzielen. Bessere Technik und Geduld bei der mikroskopischen Untersuchung der gefärbten Strichpräparate ist für die Steigerung der Auffindungshäufigkeit verantwortlich zu machen.

Nun entsteht die Frage, ob man mikroskopisch $100 \%$ positive Resultate bei allen an Neirentuberkulose Erkrankten erhalten kann. Es war nicht möglich, mikroskopisch Tuberkelbazillen bei 16 Fällen (14\%) und zwar bei 9 Fällen tuberkulöser Pyonephrose, einer Kittniere, 4 Fällen käsigkavernöser Form und bei 2 Nichtoperierten festzustellen. Unter diesen 16 Fällen konnten bei 7 Fällen tuberkulöser Pyonephrose auch kulturell keine Kolonien beobachtet werden, während sie bei den übrigen $9 \mathrm{Fällen}$ nur in geringer Zahl auftraten. Bei den ebengenannten 7 Fällen, bei denen die Bazillen weder mikroskopisch noch kulturell feststellbar waren, fand ich auch nur spärliche oder keine Kolonien bie Kultivierung aus den direkt nach der Nephrektomie durch Punktion entnommenen Nierenbeckeninhalten (näheres darüber später! Siehe Tabelle III). So bemerkte ich, dass sich bei Nierentuberkulose Fälle fanden, deren Harn wirklich so spärlich Tuberkelbazillen enthielt, dass sie kulturell nur spärlich, aber mikroskopisch gar nicht oder sogar durch beide Verfahren nicht nachweisbar waren.

Nach Wild bolz kann der Harn trotz Vorbandenseins einer Nierentuberkulose völlig frei von Bazillen sein, wenn die einzeln erkrankte Niere von der noch gesunden Blase durch Verschluss des Ureters abgetrennt ist. Ishika wa ${ }^{9)}$ aus unserer Klinik wies darauf hin, dass der Prozentsatz der positiven Bazillenbefunde mit der Zerstörung der Nierenparenchyms abnimmt. Nun fand ich, dass die Bazillenausscheidung nicht nur bei geschlossener sondern auch bei offener Pyonephrose zuweilen sehr spärlich sein kann.
II. Untersuchungsergebnisse bei durch Ureterenkatheterismus getrennt a ufgefangenen Harnen.

Der Nierenharn konnte aus der erkrankten Seite bei 51 Fällen durch Ureterenkatheterismus aufgefangen werden. Zur Kontrolle führte ich die gleiche Untersuchungen an den Neirenbeckeninhalten 72 exstirpierter Nieren durch. Die Ergenbisse dieser beiden Untersuchungsmethoden sind 
in Tabelle II, zusammengestellt.

TABELLE II.

Die Untersuchungsergebnisse bei den getrennt aufgefangenen Nierenharnen und den Nierenbeckeninhalten der exstirpierten Nieren.

\begin{tabular}{|c|c|c|c|c|c|c|c|c|}
\hline \multirow{2}{*}{\multicolumn{2}{|c|}{ Kolonienzahl }} & \multicolumn{7}{|c|}{ Zahl der Fälle bei Färbung } \\
\hline & & \multirow{2}{*}{$\frac{H}{22}$} & \multirow{2}{*}{$\frac{H}{10}$} & \multirow[t]{2}{*}{+} & \multirow[t]{2}{*}{ \pm} & \multirow[t]{2}{*}{-} & \multicolumn{2}{|c|}{ total } \\
\hline \multirow{6}{*}{ 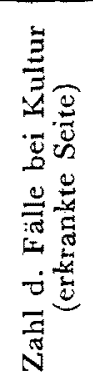 } & unzählbar & & & & & & 32 & \multirow{3}{*}{48} \\
\hline & ca. $10-50$ & & 1 & 2 & 3 & 3 & 9 & \\
\hline & $1-5$ & & 4 & 1 & & 2 & 7 & \\
\hline & - & & & & & 3 & \multicolumn{2}{|c|}{3} \\
\hline & \multirow{2}{*}{ total } & 22 & 15 & 3 & 3 & \multirow[b]{2}{*}{8} & \multirow{2}{*}{\multicolumn{2}{|c|}{51}} \\
\hline & & \multicolumn{4}{|c|}{43} & & & \\
\hline \multirow{6}{*}{ 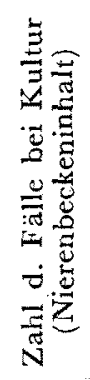 } & unzählbar & 36 & 17 & 5 & 1 & & 59 & \multirow{3}{*}{70} \\
\hline & ca. $10-50$ & & & 1 & 4 & 3 & 8 & \\
\hline & $1-5$ & & & & & 3 & 3 & \\
\hline & - & & & & & 2 & & 2 \\
\hline & \multirow{2}{*}{ total } & 36 & 17 & 6 & 5 & \multirow[b]{2}{*}{8} & & \multirow{2}{*}{72} \\
\hline & & & & & & & & \\
\hline \multirow{6}{*}{ 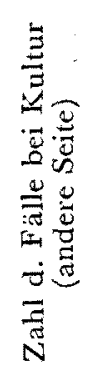 } & unzählbar & & 1 & 2 & & & 3 & \multirow{3}{*}{33} \\
\hline & ca. $10-50$ & & 2 & & 2 & 5 & 9 & \\
\hline & $1-5$ & 1 & 1 & 1 & 3 & 15 & 21 & \\
\hline & 一 & & & & 1 & 51 & & 52 \\
\hline & \multirow{2}{*}{ total } & 1 & 4 & 3 & 6 & \multirow[b]{2}{*}{71} & & \multirow[b]{2}{*}{85} \\
\hline & & & & & & & & \\
\hline
\end{tabular}

Bei allen 51 mittels Ureterenkatheter untersuchten Fällen fand ich in den Harnsedimenten der erkrankten Nierenseite stets mässig zahlreiche oder zahllose Eiterkörperchen. Die Tuberkelbazillen konnten darin mikroskopisch bei 43 Fällen (84\%) und zwar bei 37 mässig oder reichlich, bei 6 spärlich oder ganz spärlich, und kulturell bei $48(94 \%)$, und zwar bei 32 sehr reichlich, bei 16 mässig bis spärlich festgestellt werden.

$\mathrm{Watanabe}{ }^{10)}$ hat früher mitgeteilt, dass er die beiderseitigen 
Nierenharne durch Ureterenkatheterismus bilateralis bei 66 von 100 Nierentuberkulose-Fällen auffangen und mikroskopisch und kulturell die Tuberkelbazillen in dem Nierenharn der erkrankten Seite bei 38 Fällen $(58 \%$ ) feststellen konnte. Es war eine schwierige und mähsame Arbeit, sie in den getrennten Nierenharnen festzustellen, weil es schwierig ist, genügend grosse Mengen Nierenharn durch Ureterenkatheterismus aufzufangen. Nicht so häufig war es früher auch bei unserer Klinik möglich, die Bazillen in den getrennten Nierenharnen mikroskopisch nachzuweisen. Jetzt ist es mir gelungen, mikroskopisch $84 \%$ und kulturell $94 \%$ positive Bazillenbefunde aus den getrennten Nierenharnen der erkrankten Seite, also positive Resultate fast so häufig wie aus Blasenharnen und Nierenbeckeninhalten exstirpierter tuberkulöser Nieren zu erhalten. Dies ist einersieits darauf zurückzuführen, dass neuerdings das färberische, andererseits aber auch das kulturelle Verfahren sehr verbessert worden ist.

Die Kolonienzahl entsprach bei allen Fällen im grossen und ganzen der mikroskopisch gefundenen Bazillenzahl; d.h. sie waren reichlich entwickelt bei denjenigen Harmportionen, bei denen mikroskopisch reichlich Bazillen gefunden wurden und spärlich bei den mit spärlich im Strich feststellbaren Bazillen.

Die Ergebnisse aus Nierenharnen stimmte bei allen Fällen meistenteils mit den Befunden der Nierenbeckeninhalte der betreffenden exstirpierten Nieren überein: Bemerkenswert war es hier auch, dass bei einigen Fällen von Pyonephrose die Bazillen in den Nierenharnen und Nierenbeckeninhalten mikroskopisch und kulturell kaum oder nur in geringer Zahl festgestellt werden konnten. Diese Ergebnisse der Nierenharne der erkrankten Seite stimmten ausserdem grösstenteils auch mit den Blasenhambefunden fast überein.

Es ist immer leicht eine erkrankte Seite bei Nierentuberkulose festzustellen, aber immer schwierig festzustellen, ob die anderseitige Niere wirklich gesund ist. Zu diesem Zwecke dient der Ureterenkatbeterismus, und ist vor allem Untersuchung auf Eiterkörperchen und Bazillen bei den dadurch getrennt aufgefangenen Nierenharnen sehr wichtig. Unter meinen 114 an Nierentuberkulose Erkrankten wurde der scheinbar gesundseitige Nierenharn bei 85 Fällen untersucht. Unter den übrigen 29 Fällen gelang der Ureterenkatheterismus bei 10 Fällen nicht, weil die Blase oder die Uretermündung stark verändert war, und wurde bei den sonstigen 19 Fällen nicht ausgeführt, da deutliche Anzeichen einer beiderseitigen Nierenerkrankung oder einer extrarenalen schweren Komplikation schon sonst deutlich erkennbar waren.

Wie Tabelle III zeigt, war der Nierenharn völlig frei von Eiterkörperchen bei 26 gesundseitigen Portionen $(31 \%)$ von insgesammt 85 Fällen und zeigte bei den anderen 26 Fällen Leukozyten bzw. Eiterkörperchen 
bald ganz spärlich, bald vereinzelt zusammen mit zahllosen Erythrozyten, was nicht als pathologisch, sondern als normale den Erythrozyten entsprechende Leukozytenzahl aufgefasst wurde. Bei den anderen 19 Fällen wurden Eiterkörperchen spärlich und bei den übrigen 14 mässig bis zahllos gefunden. Die Harnsedimente der letzten 14 Fälle (16\%) wurden ohne weiteres als pathologisch angesehen und diese Fälle deshalb als beiderseitige Nierentuberkulose festgestellt. Es war fraglich bei meinen 19 Fällen mit spärlichen Eiterkörperchen und bei 26 mit ganzspärlichen Leukozyten bzw. Eiterkörperchen in den scheinbar gesundseitigen Nierenharnen, also im ganzen bei 45 Fällen ( $53 \%$ ), ob deise Seite wirklich gesund sei. Wichtig ist dabei die Untersuchung auf Tuberkelbazillen in diesen Harnen. Wie Tabelle III zeigt, konnten bei $10 \mathrm{~F}$ ällen von 19 mit spärlichen Eiterkörperchen und bei 20 unter 26 mit ganz spärlichen Leukozyten bzw. Eiterkörperchen Tuberkelbazillen weder mikroskopisch noch kulturell nachgewiesen werden.

\section{TABELLE III.}

Die Beziehung der Tuberkelbazillen zu Eiterkörperchen an sog. gesundseitigen Nierenharnen.

Bemg. Als ich eine bis einige Eiterkörperchen oder Leukozyten in einem jeden Gesichtsfeld sah, bezeichenete ich es ,, spärlich," und als ich einige in mehreren Gesichtsfeld sah, bezeichnete ich ,ganz spärlich."

\begin{tabular}{c|c|c|c|c|c}
\hline Bazillen & Eahleich & spärlich & $\begin{array}{r}\text { ganz } \\
\text { spärlich }\end{array}$ & negativ & total \\
\hline positiv & 13 & 9 & 6 & 6 & 34 \\
\hline negativ & 1 & 10 & 20 & 20 & 51 \\
\hline total & 14 & 19 & 26 & 26 & 85
\end{tabular}

Bezüglich der mikroskopisch sowie kulturell festgestellten Bazillengehalte in diesseitigen Nierenharnen vergleiche man Tabelle II! Sie waren bei beiden Verfahren meist nur gering, obwohl mikroskopisch bei $14(16 \%)$ und kulturell bei $33(39 \%)$ Fällen Bazillen festgestellt wurden. Vor allem betrug die Kolonienanzahl bei 21 Fällen von 33 mit kulturell positiven Bazillenbefunden weniger als 5, meistens nur 1 bis 2 .

Wie soll ich solche Fälle mit spärlichen Eiterkörperchen oder mit spärlichen Tuberkelbazillen in den anderseitigen Nierenharnen bei scheinbar einseitiger Nierentuberkulose deuten? Nach Wildbolz können spärliche Eiterkörperchen bzw. Leukozyten bald durch Ureterenkatheterismus aus der Blase in den Ureter eingeschleppt werden, 
bald aus der gesunden aber durch den Katheter momentan mechanisch gereizten Schleimhaut durchwandern und dem Ureterharn beigemischt ausgeschieden werden, obwohl die Leukozyten beim letzteren Fall immer vereinzelt, nie in Grüppchen zusammengeballt erscheinen.

Wenn die spärlichen Eiterkörperchen bei echter einseitiger Nierentuberkulose durch Ureterenkatheterismus aus der Blase in den anderseitigen Nierenharn einschleppt würden, würden auch die Tuberkelbazillen dabei mitgeführt und kämen dabei in dem eigentlich gesunden Nierenharn mit zum Vorschein. Die Einschleppung der Bazillen aus der Blase wurde bei unseren Fällen nicht immer negiert, weil der Ureterenkatheterismus bei uns ohne ,, immer fliessende Vorrichtung " ausgeführt wurde. Weil ich zahlreiche Kolonien nur bei 3 Fällen unter 33 sog. gesundseitigen Niererharnen mit kulturell positiven Befunden und nur spärliche, weniger als 5 Kolonien in der Mehrzahl der übrign Fälle feststellen konnte, während zahlreiche oder zahllose Kolonien in der Mehrzahl der krankseitigen Nierenharne gesehen wurden, und ausserdem die Bazillenkulturen bei 6 Nierenharnen ohne Eiterkörperchen positiv ausfiel, konnte ich die nicht seltene Einschleppung der Bazillen aus der Blase in die Nierenharne nicht ausschliessen. Zur Lösung dieser Frage ist es jedoch nötig, bei allen Fällen die klinischen Befunde und die Ergebnisse der verschiedenen urologischen Untersuchungen zu berücksichtigen. Ausserdem kommt hier Bazillurie ${ }^{11}$ in Frage, besonders beim Vorkommen der Tuberkelbazillen in Nierenharnen ohne Eiterkörperchen. Bei dieser Frage darf man also nur vorsichtig Schlüsse ziehen.

Unter meinen 85 Fällen fand ich nur $20(24 \%)$, bei denen ich weder Eiterkörperchen noch Bazillen in Nierenharn einer Seite finden konnte, so dass Einseitigkeit der Erkrankung bei diesen sicher war (siehe Tabelle III). Bei anderen 20 Fällen, bei denen sehr spärliche Leukozyten bzw. Eiterkörperchen aber keine Bazillen feststellbar waren, war einseitige Nierentuberkulose höchstwahrscheinlich. Schliesslich war die Diagnose der Einseitigkeit fraglich bei den übrigen 1.2 Fällen $(14 \%)$, bei denen Eiterkörperchen sehr spärlich oder nie und die Bazillen spärlich in den anderseitigen Nierenharnen nachgewiesen werden konnten, und sonst keine nennenswerte Veränderungen und Störungen durch die sonstigen urologischen Untersuchungen festgestellt wurden. Beiderseitige Tuberkulose war bei 19 Fällen $(22 \%)$ mit spärlichen Eiterkörperchen und mit oder ohne positive Bazillenbefund wahrscheinlich und bei den übrigen $14 \mathrm{~F}$ ällen (siehe Tabelle III) sicher.

Sehr bemerkenswert war es, dass ich bei Nierentuberkulose verhältnismässig wenig Fälle sah, bei denen eine einseitige Erkrankung sicher festgestellt werden konnte. Bei den meisten Fällen einseitiger Nierentuberkulose musste ich mich damit begnügen, dass die andere 
Seite relativ gesund sein sollte. Bei den oben zitierten 66 Fällen von W a t $\mathrm{n}$ a be wurden Tuberkelbazillen in anderseitigen Nierenharnen einseitiger Nierentuberkulose mikroskopisch oder kulturell nur bei 2 Fällen

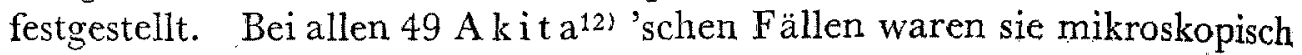
in dn sog. gesundseitigen Nierenharnen nie festgestellt, während" ganz spärliche Eiterkörperchen darin bei 34 Fällen gesehen wurden. Doch vermute ich, dass die Feststellungshäufigkeit der Tuberkelbazillen in den getrennten Nierenharnen der anderen Seite von sog. einseitiger Nierentuberkulose durch Verbesserung der Färbungsmethode und des kulturellen Verfahrens noch zunehmen wird. Dann würde die echt einseitige Nierentuberkulose viel geringer sein als man bisher gedacht hat.

\section{Schluss.}

1) Tuberkelbazillen wurden im Blasenharn mikroskopisch bei 98 Fällen $(86 \%)$ und kulturell bei $107(94 \%)$ von allen von mir untersuchten 114 Nierentuberkulose-Fällen festgestellt. Bemerkt werden muss, dass die Tuberkelbazillen bei tuberkulöser Pyonephrose so spärlich ausgeschieden werden konnten, dass sie mikroskopisch sowie kulturell schwer oder kaum nachweisbar waren, was anderseits aus meinen Untersuchungsergebnissen von Nierenbeckeninhalten exstirpierter Nieren bestätigt wurde.

2) Mit der jetzigen verbesserten Technik der Bazillenfärbung und -kultur wurden die Tuberkelbazillen in den getrennten Nierenharnen der erkrankten Seite so häufig wie in den Blasenharnen und in den anderseitigen Nierenharnen nicht selten, jedoch nur in geringer Zahl, festgestellt. So bemerkte ich, dass es bei Nierentuberkulose zuweilen schwierig war, genau festzustellen, ob die anderseitige Niere einer sog: einseitigen Nierentuberkulose wirklich gesund sei.

Diese Untersuchungen wurden mit finanzieller Unterstützüng des Unterrichtsministeriums für wissenschaftliche Forschung ausgeführt, wofür ich meinen herzlichsten Dank ausspreche. Prof. M. Muto

\section{Literatur.}

(1) Kon, Tohoku Igaku Zassi, 1944, 34, 511.

(2) Oka, Nippon .Rinsho-Kekkaku, 1940, 1, 829.

(3) Wild bolz, Handbuch der Urologie, Berlin, 1927, 4, 1.

(4) Takahasi, u. a., Hifuka-Hinyokika Zassi, 1939, 46, 256.

(5) Koi zumi, Tohoku Igaku Zassi, 941, 29, 288.

(6) Y a m ad a, Nippon Hinyokibyo-Gakkai Zassi; 1941, 31, 321.

(7) Tomikawa, Zitti-Ika to Rinsho, 1942, 19, 833.

(8) Kitag a wa u. a., Nippon-Ikadaigaku Zassi, 1940, 11, 1131. 
(9) Ishikawa, Mitt. altg. Path. u. Anat., 1937, 9, 315.

(10) Wa ta na be, Nippon Hinyokibyo-Gakkai Zassi, 1930, 19, 485.

(11) Wildbolz, M. m. W., 1934, 81, 1012.

(12) Akita, Nippon Hinyokibyo-Gakkai Zassi, 1930, 19, 712. 